\title{
Thrombocytosis in an infant with a TRPV4 mutation: a case report
}

Christopher S Thom, ${ }^{1}$ Erik Brandsma, ${ }^{1}$ Michele P Lambert ${ }^{2}$

${ }^{1}$ Division of Neonatology, Children's Hospital of Philadelphia, Philadelphia, PA, USA

${ }^{2}$ Division of Hematology, Children's Hospital of Philadelphia, Philadelphia, PA, USA

Corresponding Author:

Christopher S Thom

Children's Hospital of Philadelphia

3401 Civic Center Blvd

2NW46, Division of Neonatology

Philadelphia, PA 19146

thomc@email.chop.edu

Tel: $267-760-7684$

Fax: 888-719-1759

Running Title: Thrombocytosis and TRPV4 mutation 


\title{
Thrombocytosis in an infant with TRPV4 mutation: a case report
}

\author{
Mutations in the calcium channel gene Transient Receptor Potential \\ cation channel subfamily V member 4 (TRPV4) cause autosomal dominant \\ skeletal dysplasia, with phenotypes ranging from mild to perinatal \\ lethality. A recent report detailed murine thrombocytosis in the context of \\ pharmacologic TRPV4 inhibition, but no prior reports have described \\ platelet count abnormalities in the context of human TRPV4 disease. Here, \\ we report a case of prolonged thrombocytosis in the context of TRPV4- \\ associated metatropic dysplasia that was lethal in the infantile period.
}

Keywords: thrombocytosis; skeletal dysplasia; TRPV4; calcium channel

\section{Introduction}

Mutations in TRPV4 (Transient Receptor Potential cation channel subfamily V member 4) cause a clinically heterogenous metatropic skeletal dysplasia with autosomal dominant inheritance. ${ }^{1,2}$ Metatropic dysplasia is characterized by short extremities, short trunk, progressive kyphoscoliosis, and distinct craniofacial abnormalities. Phenotypic severity ranges from mild to perinatal lethal, with death typically due to thoracic insufficiency. ${ }^{1,2}$ Skeletal manifestations arise from altered TRPV4 calcium sensing and perturbed chondrocyte differentiation. ${ }^{2}$

In a cellular model, TRPV4 inhibition impacted proplatelet formation. ${ }^{3}$ In vivo, megakaryocytes sensed decreased extracellular matrix stiffness, increasing PI3K/AKT pathway activation and platelet count. ${ }^{3}$ To our knowledge, no prior reports have described altered platelet counts in humans with metatropic dysplasia.

Here, we report a case of TRPV4 mutation with lethality around 4 months of age. The infant had lifelong thrombocytosis without known associated clinical complications directly related to the platelet count. This is the first report of thrombocytosis in human TRPV4-associated metatropic dysplasia. Thrombocytosis might be also observed in similar clinical cases.

\section{Case}

Prenatal testing for the female patient was consistent with arthrogryposis, including decreased thoracic circumference, spinal segmentation anomalies, thoracolumbar kyphoscoliosis, lumbosacral lordosis, and a tethered spinal cord. Upper and lower extremities were noted to have shortened long bones with concerns for contracture, with lower extremities more severely affected. MRI showed no significant structural anomalies of the brain or spine.

The patient was born at 32 weeks 1 day gestation by cesarean section, in the setting of prolonged premature rupture of membranes and recurrent fetal decelerations. She was admitted to our Level 4 neonatal and infant intensive care unit for respiratory support and multidisciplinary evaluation. Her clinical exam was consistent with the described prenatal imaging findings. Clinical exome sequencing identified a heterozygous de novo variant in TRPV4 (c.1303 G>A, p.E435K). This mutation was not previously reported in the ClinVar or Human Mutation Gene Database registries, but was suspected to be 
pathogenic (SIFT scores 0.02-0.03 rated as 'deleterious', PolyPhen scores 0.141-0.767 rated from 'benign' to 'possibly damaging').

The patient required non-invasive support via continuous positive airway pressure (CPAP), bilevel positive airway pressure (BIPAP) or high flow nasal cannula for her entire life. She was intubated in the setting of procedures, including a laparoscopic gastrostomy tube placement and fundoplication at 3 months of age. At almost 4 months of age, the infant acutely decompensated in the setting of abdominal compartment syndrome. She was unable to be resuscitated despite emergent exploratory laparotomy. The cause of this abdominal catastrophe was unknown, despite full autopsy.

Thrombocytosis was noted throughout the infant's life, with a maximum recorded platelet count more than 3 times the upper limit of normal $(1,247,000$ per $\mu 1$, Figure 1). There were no other consistently abnormal hematologic parameters on complete blood counts (Table 1). Thrombocytosis was unexpected, as platelet count derangement had not been previously described in clinical descriptions of TRPV4-related disease.

Though the etiology remained unclear despite subspecialist discussions, there was never an indication for intervention, treatment or other alteration in clinical management as a result of the high platelet count alone. The patient underwent one sepsis evaluation, which was triggered by acute respiratory decompensation where thrombocytosis was noted as evidence supporting the use of empiric antibiotics to rule out possible bacterial infection. When her blood culture remained negative after 48 hours, antibiotics were discontinued.

\section{Discussion}

TRPV4 mutations cause autosomal dominant, life-limiting metatropic dysplasia.

Clinical descriptions of the related skeletal manifestations are well documented, ${ }^{1,2}$ as are potential associated clinical neuromuscular ${ }^{4,5}$ and neuropathic ${ }^{6,7}$ manifestations. To our knowledge, aberrancies in hematologic parameters have not previously been reported in association with human TRPV4 mutation. However, TRPV4 inhibition was recently linked to increased platelet production in mice. ${ }^{3}$

Benign thrombocytosis may be a clinical component of TRPV4-associated metatropic dysplasia, although it is impossible to extrapolate a generalized or mechanistic association between TRPV4 mutation and thrombocytosis from a single clinical case. The thrombocytosis observed in this case may have resulted from altered extracellular matrix stiffness and megakaryocyte reactivity, in agreement with cellular and murine phenotypes. ${ }^{3}$ However, we cannot exclude chronic inflammation or respiratory support as potential confounders. Reactive thrombocytosis can occur in the setting of inflammation ${ }^{8}$ or lung injury. ${ }^{9}$ Indeed, platelet count was within the normal range for the first few days of our patient's life (Fig. 1), although premature rupture of membranes and fetal distress at the time of birth may confound interpretation of these initial platelet count values. A small focus of extramedullary hematopoiesis was identified on autopsy of the lung in this patient, but we suspect that this was most likely an incidental finding of limited clinical consequence.

To validate the clinical association of platelet count and TRPV4 mutation, it will be important to investigate clinical phenotypes associated with other TRPV4 mutation 
cases. It is possible that specific mutations, or functional TRPV4 perturbations, could be linked to altered platelet count. Indeed, TRPV4 mutations can lead to a spectrum of clinical phenotypes. ${ }^{1}$

As an acute phase reactant, thrombocytosis can be a sign of infection, inflammation or other pathology. ${ }^{8}$ In some clinical scenarios, particularly in critically ill neonates, thrombocytosis can lead to sepsis workups and/or other invasive procedures. As such, recognition of conditions that can cause thrombocytosis is important. In the reported case, thrombocytosis did not cause known clinical complications and was not associated with acute pathology. We hope that presenting this case may inform diagnostic workup in similar clinical cases. However, until a link between TRPV4 mutation and thrombocytosis can be confirmed in other cases or case series, our report should not preclude or deter a detailed workup related to thrombocytosis in similar cases.

There was prognostic uncertainty in this case of TRPV4-associated metatropic dysplasia, although the prognosis was guarded based on respiratory support needs early in life. It might also be difficult to predict disease severity for some individuals with TRPV4 variants of unknown significance. Platelet count might act as a non-invasive biomarker for TRPV4 function and/or disease severity in the right clinical context. It will be important for future studies to determine whether thrombocytosis correlates with TRPV4-associated disease severity. 


\section{Methods}

Ensembl variant effect predictor software was used to calculate SIFT and PolyPhen scores related to this variant (https://useast.ensembl.org/Tools/VEP).

Ethics: The Children's Hospital of Philadelphia Institutional Review Board deemed this study (IRB 20-017245) exempt from oversight on January 23, 2020.

\section{Acknowledgements}

We thank Alexandra Pomar, MSW, LSW for her assistance. We thank the family for their willingness to share this story.

\section{Contributions}

CST, EB, and MPL interpreted data and contributed to writing this manuscript.

\section{Funding}

This work was funded through T32HD043021 (CST) and an American Academy of Pediatrics Marshall Klaus Neonatal-Perinatal Research Award (CST).

\section{Disclosures of interest}

The authors declare no relevant conflicts of interest.

\section{Consent}

The patient's family has given written consent to the inclusion of material pertaining to the patient's case. They acknowledge that they cannot be identified via the paper, as the patient has been fully anonymized in this manuscript. 


\section{References}

1. Andreucci, E. et al. TRPV4 related skeletal dysplasias: A phenotypic spectrum highlighted byclinical, radiographic, and molecular studies in 21 new families. Orphanet J. Rare Dis. 6, (2011).

2. Camacho, N. et al. Dominant TRPV4 mutations in nonlethal and lethal metatropic dysplasia. Am. J. Med. Genet. Part A 152, 1169-1177 (2010).

3. Abbonante, V. et al. A new path to platelet production through matrix sensing. Haematologica 102, 1150-1160 (2017).

4. Auer-Grumbach, M. et al. Alterations in the ankyrin domain of TRPV4 cause congenital distal SMA, scapuloperoneal SMA and HMSN2C. Nat. Genet. 42, 160-164 (2010).

5. Biasini, F. et al. TRPV4 related scapuloperoneal spinal muscular atrophy: Report of an Italian family and review of the literature. Neuromuscul. Disord. 26, 312315 (2016).

6. McEntagart, M. TRPV4 axonal neuropathy spectrum disorder. Journal of Clinical Neuroscience 19, 927-933 (2012).

7. Zimoń, M. et al. Dominant mutations in the cation channel gene transient receptor potential vanilloid 4 cause an unusual spectrum of neuropathies. Brain 133, 1798-1809 (2010).

8. Klinger, M. H. F. \& Jelkmann, W. Role of blood platelets in infection and inflammation. J. Interf. Cytokine Res. 22, 913-922 (2002).

9. Weyrich, A. S. \& Zimmerman, G. A. Platelets in Lung Biology. Annu. Rev. Physiol. 75, 569-591 (2013). 


\section{Figure}

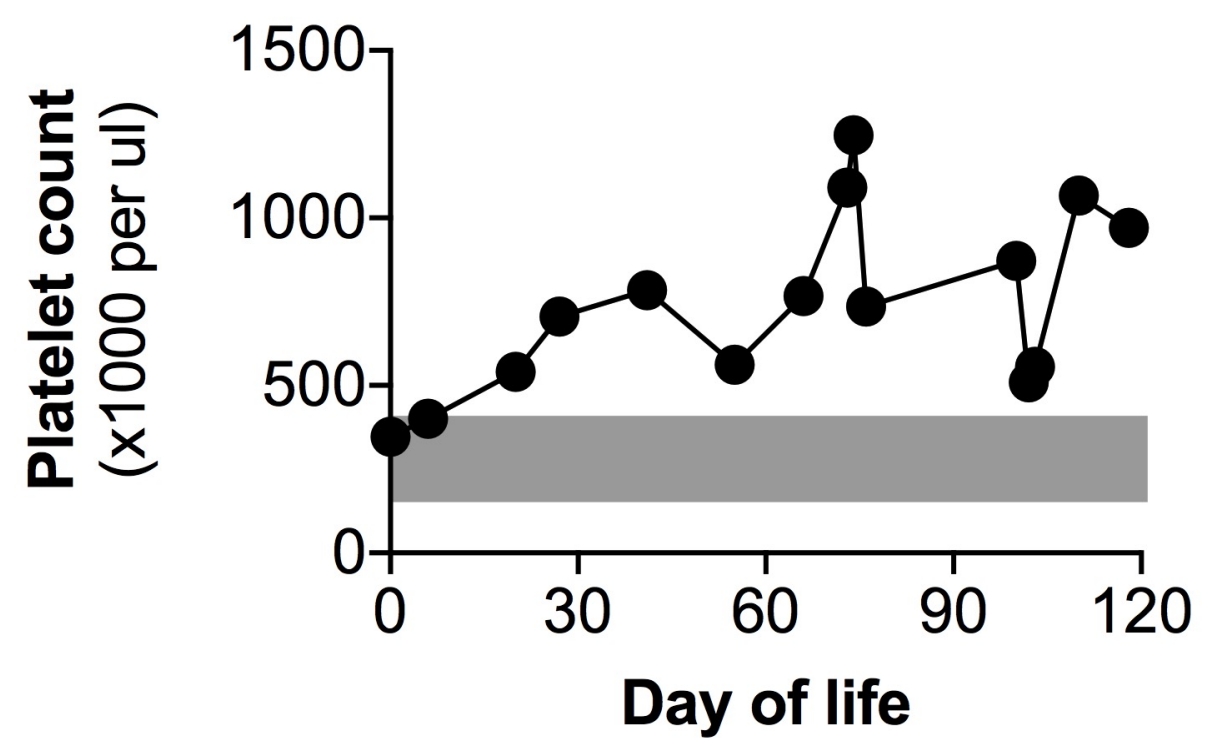

Figure 1. Recorded platelet counts in this patient. Gray zone indicates normal reference range $(150-400 \times 1000$ platelets per $\mu l)$. 


\section{Table}

Table 1. Hematologic trait values for this patient with associated normal reference ranges. Shown are the full range of laboratory values obtained, as well as the mean \pm standard deviation for each parameter ( $\mathrm{n}=15$ complete blood counts). PLT, platelet count. MPV, mean platelet volume. RBC, red blood cell count. Hb, hemoglobin. Hct, hematocrit. $\mathrm{MCV}$, mean corpuscular volume. $\mathrm{MCH}$, mean corpuscular hemoglobin.

$\mathrm{MCHC}$, mean corpuscular hemoglobin concentration. RDW, red cell distribution width. WBC, white blood cell count.

\begin{tabular}{|llcc|} 
Trait & Normal Range & Range & Mean \pm SD \\
\hline $\mathrm{PLT}$ & $150-400 \times 1000 / \mathrm{ul}$ & $347-1247$ & $743 \pm 266$ \\
\hline $\mathrm{MPV}$ & $9.0-10.9 \mathrm{fL}$ & $8.4-11.1$ & $9.3 \pm 0.9$ \\
\hline $\mathrm{RBC}$ & $3.1-4.5 \times 10^{\wedge} 6 / \mathrm{ul}$ & $3.3-5.2$ & $4.1 \pm 0.6$ \\
\hline $\mathrm{Hb}$ & $9.5-13.5 \mathrm{~g} / \mathrm{dL}$ & $10.2-14.8$ & $12.1 \pm 1.8$ \\
\hline $\mathrm{Hct}$ & $29-41 \%$ & $30.3-44.8$ & $35.9 \pm 5.1$ \\
\hline $\mathrm{MCV}$ & $74-108 \mathrm{fL}$ & $81.5-100.5$ & $87.8 \pm 5.2$ \\
\hline $\mathrm{MCH}$ & $25-35 \mathrm{pg}$ & $26.6-36.0$ & $29.7 \pm 2.7$ \\
\hline $\mathrm{MCHC}$ & $30 .-36 \mathrm{~g} / \mathrm{dL}$ & $31.1-36.5$ & $33.8 \pm 1.3$ \\
\hline $\mathrm{RDW}$ & $35.2-45.1 \mathrm{fL}$ & $35.9-61.0$ & $44.1 \pm 8.1$ \\
\hline $\mathrm{WBC}$ & $6.0-13.3 \times 1000 / \mathrm{ul}$ & $7.7-29.6$ & $14.7 \pm 5.3$ \\
\hline
\end{tabular}

